\title{
A novel Osmium-based compound targets the mitochondria and triggers ROS-dependent apoptosis in colon carcinoma
}

\author{
A Maillet ${ }^{1}, \mathrm{~S}$ Yadav $^{1}, \mathrm{YL} \mathrm{Loo}{ }^{1}, \mathrm{~K}$ Sachaphibulkij ${ }^{1}$ and S Pervaiz ${ }^{\star, 1,2,3,4}$
}

Engagement of the mitochondrial-death amplification pathway is an essential component in chemotherapeutic execution of cancer cells. Therefore, identification of mitochondria-targeting agents has become an attractive avenue for novel drug discovery. Here, we report the anticancer activity of a novel Osmium-based organometallic compound (hereafter named Os) on different colorectal carcinoma cell lines. HCT116 cell line was highly sensitive to Os and displayed characteristic features of autophagy and apoptosis; however, inhibition of autophagy did not rescue cell death unlike the pan-caspase inhibitor z-VADfmk. Furthermore, Os significantly altered mitochondrial morphology, disrupted electron transport flux, decreased mitochondrial transmembrane potential and ATP levels, and triggered a significant increase in reactive oxygen species (ROS) production. Interestingly, the sensitivity of cell lines to 0 s was linked to its ability to induce mitochondrial ROS production (HCT116 and RKO) as HT29 and SW620 cell lines that failed to show an increase in ROS were resistant to the death-inducing activity of Os. Finally, intra-peritoneal injections of Os significantly inhibited tumor formation in a murine model of HCT116 carcinogenesis, and pretreatment with Os significantly enhanced tumor cell sensitivity to cisplatin and doxorubicin. These data highlight the mitochondria-targeting activity of this novel compound with potent anticancer effect in vitro and in vivo, which could have potential implications for strategic therapeutic drug design.

Cell Death and Disease (2013) 4, e653; doi:10.1038/cddis.2013.185; published online 6 June 2013

Subject Category: Cancer

Recent evidences indicate that most chemotherapeutic agents trigger death execution in cancer cells; however, the phenotype of death may vary from classical apoptosis to autophagy, necrosis or necroapoptosis. ${ }^{1}$ Despite advances in the therapeutic management of a host of cancers, chemotherapy resistance remains a major challenge due to acquired mutations or deletions in specific genes regulating these cell death pathways. Therefore, there is a need to develop novel compounds that could efficiently engage execution pathways for targeted deletion of cancer cells. In this regards, the central role of mitochondria in mediating numerous physiological pathways and especially programmed cell death has led to an interest in exploiting them as targets for anticancer therapies. $^{2-5}$ Mitochondria have an obligatory role in the activation of the apoptosome during intrinsic death signaling, compared with the extrinsic pathway where caspases' activation is initiated by cell surface death receptors. ${ }^{6,7}$ This is brought about by an increase in mitochondrial outer membrane permeability (MOMP), with the resultant egress of apoptogenic proteins such as cytochrome $c$, apoptosis-inducing factor (AIF) or Smac/Diablo from the inter-membranous space to the cytosol. ${ }^{8}$ Once in the cytosol, cytochrome $c$ is able to conjugate to the apoptosis protease-activating factor 1 (Apaf-1) in the presence of ATP, and thus activate procaspase 9. In turn, active caspase 9 activates the downstream caspase cascade that involves caspase 3 and other effector enzymes. ${ }^{9}$ In addition to the release of apoptogenic factors involved in activation of caspase-dependent and caspase-independent cell death mechanisms, mitochondrial damage and loss of vital mitochondrial functions may lead passively to cell death because of compromised energy production. ${ }^{10}$ Thus, structural or functional alterations of the mitochondria, such as dissipation of the inner membrane potential, disruption of electron transport and deregulation in oxidative phosphorylation, may directly or indirectly amplify death execution signals.

It is now well established that the redox status of the cell has a key role in cell fate. Indeed, a slight deregulation of the balance between the rates of production and breakdown of reactive oxygen and nitrogen species (ROS and RNS) can lead to the activation of cell death pathways. ${ }^{11-13}$ Of note, due to the high flux of electrons through the electron transport chain (ETC) that facilitates the leakage of electrons onto oxygen, mitochondria are a major intracellular source of ROS, mainly superoxide $\left(\mathrm{O}_{2}^{-}\right)$and hydrogen peroxide $\left(\mathrm{H}_{2} \mathrm{O}_{2}\right){ }^{14}$

\footnotetext{
${ }^{1}$ ROS, Apoptosis and Cancer Biology Laboratory, Department of Physiology, Yong Loo Lin School of Medicine, National University of Singapore, Singapore, Singapore; ${ }^{2}$ NUS Graduate School for Integrative Sciences and Engineering, National University of Singapore, Singapore, Singapore; ${ }^{3}$ Cancer and Stem Cell Biology Program, Duke-NUS Graduate Medical School, Singapore, Singapore and ${ }^{4}$ Singapore-MIT Alliance, Singapore, Singapore

${ }^{*}$ Corresponding author: S Pervaiz, ROS, Apoptosis and Cancer Biology Laboratory, Department of Physiology, Yong Loo Lin School of Medicine, National University of Singapore, 2 Medical drive, Building MD9 no. 01-05, Singapore 117597, Singapore. Tel: +65 65166602; Fax: +65 67788161; E-mail: phssp@ nus.edu.sg Keywords: cancer therapy; novel compound; mitochondria; ROS

Abbreviations: $\Delta \Psi_{\mathrm{m}}$, mitochondrial transmembrane potential; 3MA, 3 methyladenine; ETC, electron transport chain; $\mathrm{H}_{2} \mathrm{O}_{2}$, hydrogen peroxide; LC3, microtubuleassociated protein light chain 3; MOMP, mitochondrial outer membrane permeabilization; NAC, N-acetyl cysteine; NAO, nonyl acridine orange; Nox, NADPH oxidase; $\mathrm{O}_{2}^{-}$, superoxide; PARP, poly ADP ribose polymerase; PTP, permeability transition pore; RNS, reactive nitrogen species; ROS, reactive oxygen species Received 19.3.13; revised 29.4.13; accepted 30.4.13; Edited by M Agostini
} 
Therefore, excessive accumulation of ROS from extra- or intramitochondrial sources could activate and/or amplify death execution, which provides a rationale for the design and development of redox-modifying small molecule compounds. In this regard, we recently reported the critical involvement of intracellular ROS in autophagy-associated apoptosis of human cancer cells by a novel small molecule. ${ }^{15}$ Interestingly, a number of similar observations involving simultaneous induction of autophagy and apoptosis with other compounds have been reported, ${ }^{16-18}$ and the precise role of autophagy during death execution is still being debated. ${ }^{19}$

Osmium (Os), which is closely related to platinum, a commonly used heavy metal chemotherapeutic, ${ }^{20,21}$ has shown promise against a variety of cancers, including ovarian and colon cancers. ${ }^{22}$ As platinum-based compounds (such as cisplatin) are associated with untoward side effects as well as drug resistance, Os-based agents offer a new avenue for exploration. Here, we report the antitumor activity in vitro and in vivo of a novel Os-based compound on colon cancer cell lines. Results show that Os induces changes in mitochondrial morphology and function, triggers apoptosis in a ROSdependent manner and inhibits tumor growth in a murine model of colon carcinogenesis.

\section{Results}

Os induces cell death in human colorectal carcinoma cells with autophagic features. We first evaluated the death-inducing activity of Os in HCT116 HCC cells. Exposure to Os for $24 \mathrm{~h}$ resulted in a dose-dependent decrease in cell viability, with an IC50 between 50 and $75 \mu \mathrm{M}$ (Figure 1a) as well as morphological features consistent with cell loss/death (Supplementary Figure S1B). In addition, long-term survival assay revealed a highly significant reduction in tumor clonogenic ability upon incubation with different concentrations of Os (Supplementary Figure S1C). Interestingly, a closer analysis of cells indicated the presence of multiple vacuoles in cells incubated with Os, which suggested the induction of autophagy. As autophagy has been linked to cell death in a variety of model systems, we next set out to investigate whether autophagy had a central role in death induced by Os or was merely an associated event. Indeed, kinetic analysis of cells following exposure to Os showed increased conversion of the cytosolic form of microtubuleassociated protein 1 light chain LC3-I into LC3-II by western blot analysis (Supplementary Figure S2A). This increase in LC3-Il formation was downstream of ERK phosphorylation induced by Os and could be blocked by either pharmacological or genetic inhibition of ERK (Supplementary Figures S3A-C). Despite inhibiting Os-induced LC3-II formation, ERK inhibition did not have any effect on cell viability (Supplementary Figure S3D).

As the cellular level of LC3-II may not accurately reflect autophagic activity, ${ }^{23}$ the autophagic flux to the lysosomal compartment was investigated by analyzing LC3-II in cells pretreated with lysosomal inhibitors, E64D and pepstatin-A; lysosomal inhibitors should induce LC3-II accumulation by blocking the autophagosomal-lysosomal fusion. Results show that the presence of lysosomal inhibitors further increased LC3-II levels in lysates from Os-treated cells
(Supplementary Figure S2B). In addition, protein levels of p62/SQSTM1, another marker of autophagic flux, were significantly reduced following $16-24 \mathrm{~h}$ of exposure to Os (Supplementary Figure S2B). These data provide evidence for efficient autophagic flux induced by Os. To gain further insight into autophagic pathway, we looked at the expression of one of the key autophagy-mediating proteins, Beclin-1. Results indicate that Beclin-1 expression was not induced upon exposure to Os, but instead a time-dependent decrease in Beclin-1 was observed (Supplementary Figure S2A). Furthermore, siRNA-mediated gene silencing of Beclin-1 neither inhibited LC3-II formation nor rescued cells from the death-inducing effects of Os (Supplementary Figures S2C and D). Similarly, preincubation with the PI3K inhibitor, 3 methyladenine (3MA), did not block cell death induced by Os (Supplementary Figure S2E). These results indicate that a selective and noncanonical pathway of autophagy was triggered by Os in HCT116 cells, however autophagy did not contribute to Os-induced cell death.

Os triggers apoptotic death by targeting mitochondrial metabolism. As mitochondria are critical in drug-induced cell death, we next asked if the antitumor activity of Os was a function of apoptotic execution secondary to its effect on mitochondrial function. Firstly, cell cycle analysis after $24 \mathrm{~h}$ of exposure of HCT116 cells to Os showed a significant increase in the sub-G1 (sub-diploid DNA) apoptotic fraction (Figure 1b). This was further corroborated by the release of cytochrome $c$ from the mitochondria to the cytosol (Figure 1c), an indication of MOMP, as well as significant increases in caspases 9 and 3 activities (Figures 1d-f). Furthermore, Os-induced cell death was virtually completely inhibited in the presence of the pan-caspase inhibitor Z-VADfmk (Figure 1g). Interestingly, neither the overexpression of $\mathrm{Bcl}-2$ (CEM-Bcl-2 cells) nor the absence of Bax and Bak (HCT116 $\mathrm{Bax}^{-} /^{-}$and HCT Bax/Bak ${ }^{-} /^{-}$cells) had any significant effect on Os-induced cell death (Supplementary Figures S4A-E), thereby ruling out the involvement of the Bcl-2 family.

Intrigued by the findings that Bax/Bak were not involved in mitochondrial permeabilization and that overexpression of $\mathrm{Bcl}-2$ did not block cell death, we next asked whether Os could be mediating its effects via direct targeting of the mitochondrion, an obligate organelle in the apoptotic pathway. ${ }^{24,25}$ To determine whether Os leads to structural changes to mitochondria, we collected transmission electron microscopic images of HCT116 cells at different time points after Os treatment. Mitochondria from DMSO-treated HCT116 cells were of normal morphology, and showed condensed and electron-dense dark cristae; however, after incubation with Os, mitochondria appeared swollen with expanded and transparent matrices (Figure 2a). We next assessed the general metabolic activity of mitochondria by analyzing the levels of ATP in Os-treated cells at different time points. Results showed that intracellular ATP levels of drug-treated cells dropped in a time-dependent manner, reaching $50 \%$ of untreated control cells at $6 \mathrm{~h}$ post treatment (Figure $2 \mathrm{~b}$ ). Subsequently, we checked the effect of Os on different component of the ETC. Proteins from whole-cell lysates were 
a
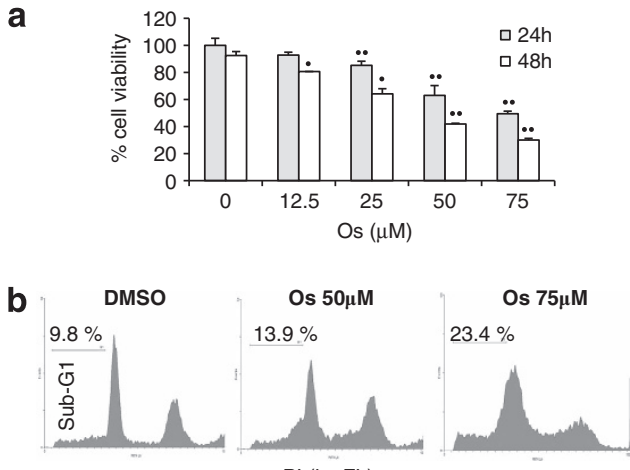

$\mathrm{PI}(\log \mathrm{FL})$
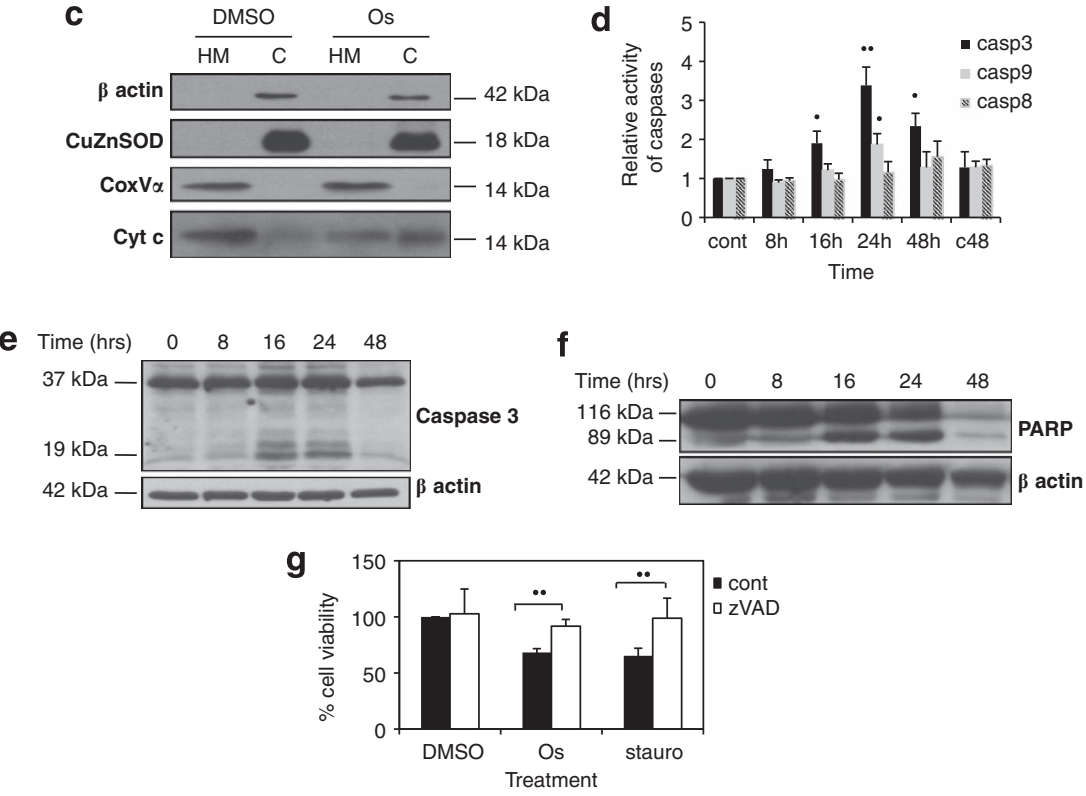

Figure 1 Os induces cell death in cancer cells via apoptosis. (a) HCT116 cells $\left(1.25 \times 10^{5}\right)$ were seeded in 24 -well plates, treated with increasing doses of Os for 24 or $48 \mathrm{~h}$ and cell survival was assessed by crystal violet assay. Results are expressed as average of the percentage of the relative growth. $\mathrm{HCT} 116 \mathrm{cells}$ were treated with $50 \mu \mathrm{M}$ of Os for $16 \mathrm{~h}$, (b) cell cycle profiles were obtained by PI staining and (c) subcellular fractions were subjected to western blot analysis. (d) Os-treated cells were harvested and assayed for caspases 3, 8 and 9 activities using fluorogenic substrates, as described in Materials and Methods. Lysates from cells treated with Os (50 $\mu \mathrm{M}$ ) were probed for processing of caspase 3 (e) and PARP cleavage (f). (g) Cells were preincubated with zVAD (100 $\mu \mathrm{M}$ for $1.5 \mathrm{~h})$ before exposure to Os $(50 \mu \mathrm{M})$ or staurosporine $(2.5 \mu \mathrm{M})$ for $24 \mathrm{~h}$, and survival was assessed by crystal violet staining. $\bullet P<0.05, \bullet P<0.025$ versus control

collected after Os treatment and ETC complexes I, II and IV activities were measured using enzymatic assays. Our results did not reveal any significant changes in the activity of complex I or IV of the ETC (data not shown); however, a decrease in complex II activity was observed (Figure 2c). In addition, there was a significant decrease in mitochondrial transmembrane potential $\left(\Delta \Psi_{\mathrm{m}}\right)$ of cells treated for 3 and $6 \mathrm{~h}$ with Os (Figure 2d).

Previous studies have demonstrated that the autophagic machinery can be involved in mitochondrial degradation and clearance of damaged mitochondria. ${ }^{26,27}$ However, if damaged mitochondria are not disposed of in time, it results in oxidation of cardiolipin, lipid localized at the inner membrane of the mitochondria and further impairment of the organelle. Oxidation of cardiolipin can be detected by flow cytometry analysis using nonyl acridine orange (NAO) that specifically interacts with nonoxidized, intact cardiolipin. Our data revealed reduced NAO fluorescence after 4 and $6 \mathrm{~h}$ of Os treatment, indicating ROS-mediated cardiolipin oxidation (Figure 2e). Taken together, these results provide evidence that Os treatment results in alteration in both mitochondrial morphology and function.

Os-induced cell death is dependent on mitochondrial ROS production. There is a growing body of evidence that generation of oxygen species (ROS) affects cell sensitivity and can lead to the activation of cell death pathways. ${ }^{28,29}$ Stimulated by the findings indicating cardiolipin oxidation in cells treated with Os, we set out to assess the production of intracellular ROS species using two different fluorescent probes (MitoSox RED and DCFH-DA). Kinetics analysis of mitochondrial $\mathrm{O}_{2}^{-}$clearly indicated the ability of $\mathrm{Os}$ to induce an increase in intramitochondrial $\mathrm{O}_{2}^{-}$levels as early as $1 \mathrm{~h}$ (Figure $3 \mathrm{a}$ and Supplementary Figure S5A) and up to $24 \mathrm{~h}$ post treatment (data not shown). Furthermore, exposure of HCT116 cells to Os also resulted in a significant increase in 
a

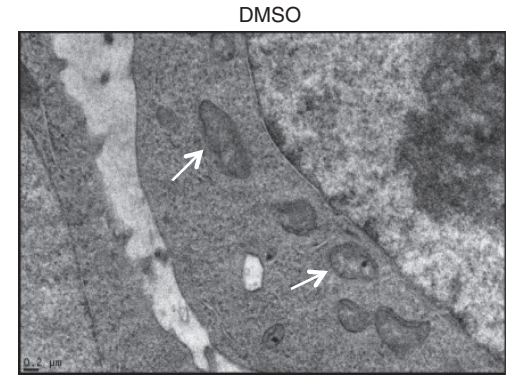

b
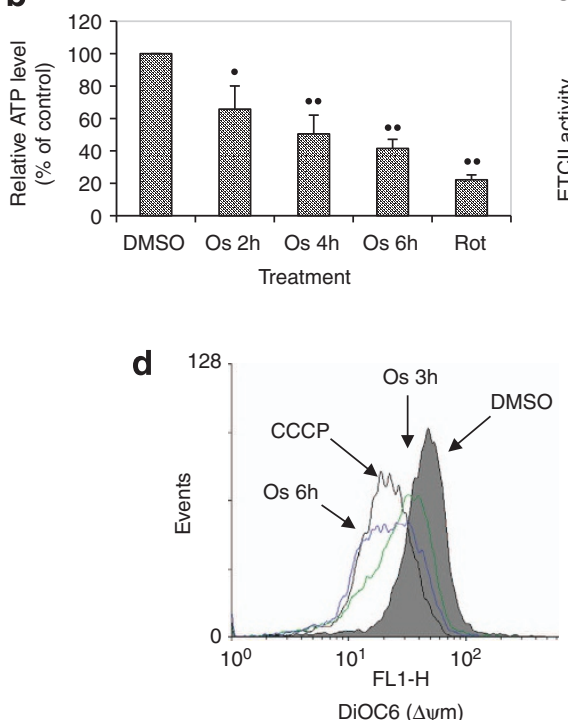

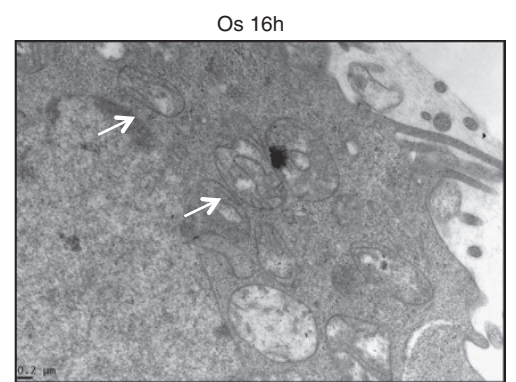

c

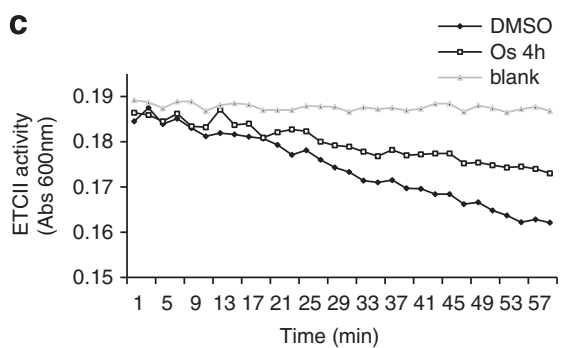

Time (min)

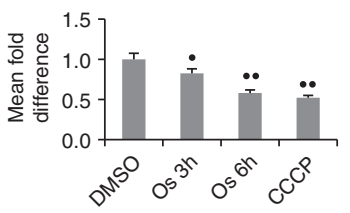

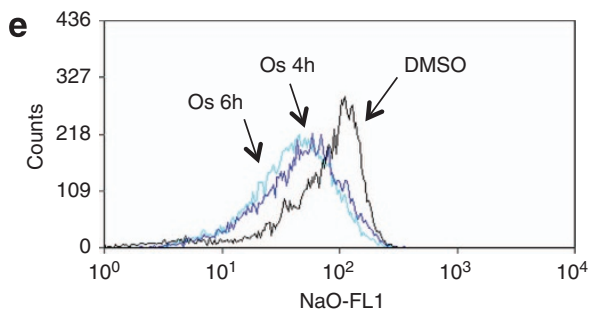

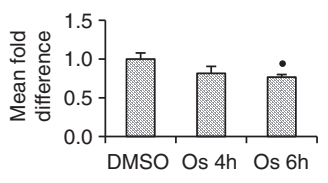

Figure 2 Os affects mitochondrial function and morphology. (a) HCT116 cells were treated with Os (50 $\mu \mathrm{M})$ for $16 \mathrm{~h}$, fixed and viewed under electron microscope (magnification $\times 25000$ ) White arrows pointing to mitochondria. (b) Relative ATP level was determined in HCT116 cells using the ATP Bioluminescence Assay Kit CLS II (Roche), as described in Materials and Methods. (c) A total of $6 \times 10^{5} \mathrm{HCT} 116$ cells were incubated with $50 \mu \mathrm{M}$ of Os for $4 \mathrm{~h}$ and ETC-II activity was determined using the Human Complex activity Microplate Assay Kit (Mitosciences). (d) Os-treated cells were incubated with the potential-sensitive probe DiOC 6 , and $\Delta \Psi_{\mathrm{m}}$ was analyzed by flow cytometry as described in Materials and Methods. Representative bar graphs were plotted with the G-mean values to determine the fold difference in $\Delta \Psi_{\mathrm{m}}$ between control and Os-treated groups. (e) Os-treated cells were loaded with $\mathrm{N}$-nonyl-acridine-orange, and cardiolipin oxidation was analyzed by flow cytometry as described in Materials and Methods. Quantification of G-mean values have been reported on the corresponding graphs. ${ }^{\bullet} P<0.05,{ }^{\bullet} P<0.025$ versus control

$\mathrm{H}_{2} \mathrm{O}_{2}$ production as measured by the DCFH-DA probe (Figure $3 b$ and Supplementary Figure S5B), which was blocked by preincubation of the cells with the ROS scavenger $\mathrm{N}$-acetyl cysteine (NAC) (Figure 3b). Pretreatment with NAC also rescued tumor cells from the short-term (24 h) and longterm (colony-forming ability) cytotoxic effects of Os (Figures $3 c-d)$. In addition, western blot analysis of total cell lysate pretreated with the ROS scavenger showed that NAC was able to decrease poly ADP ribose polymerase (PARP) cleavage, thus indicating that ROS production occurred upstream of apoptosis (Figure $3 e$ ), data that corroborate our recent findings.
Colon cancer cells that fail to produce ROS are resistant to Os-induced cell death. Stimulated by our findings linking intracellular ROS production to the death-inducing activity of Os, we next assessed the response of different colon cancer cell line to Os treatment. Firstly, we noticed that similar to HCT116 cells, RKO carcinoma cells were highly sensitive to Os-induced apoptosis. On the contrary, the adenocarcinoma cell lines, HT29 and SW620, were resistant to the short-term and long-term cytotoxic effects of Os (Figure $4 \mathrm{a}$ and Supplementary Figure S6A); caspase 3 activity was increased in RKO cells treated with Os (up to seven fold after $24 \mathrm{~h}$ ) and remained equivalent to the control in HT29 
a

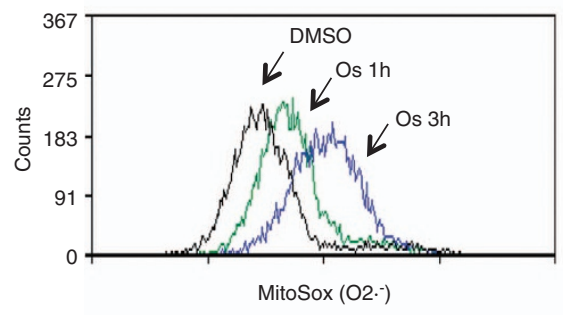

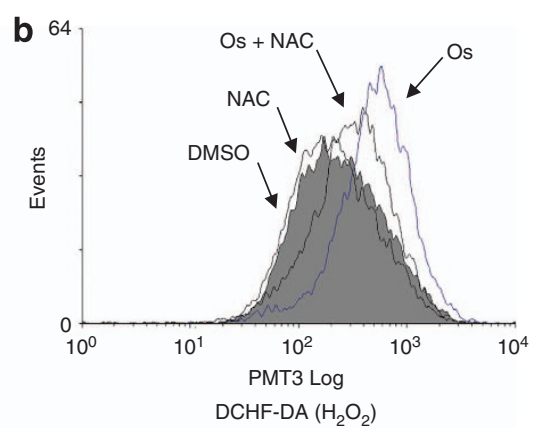

d

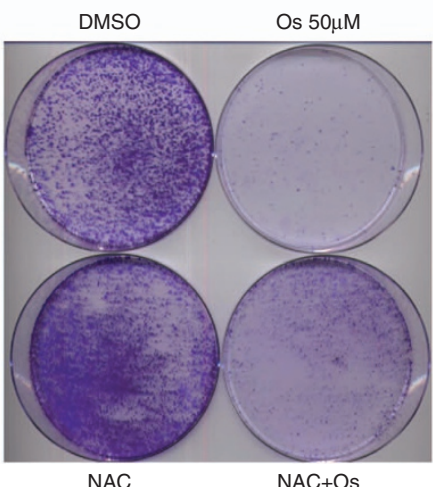

C

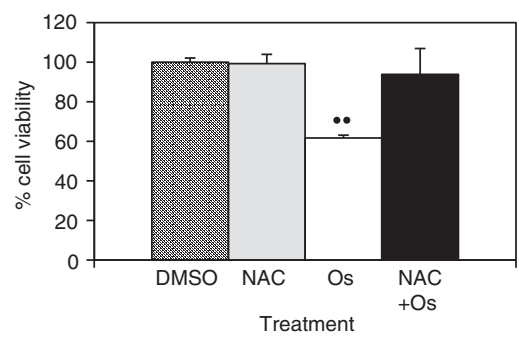

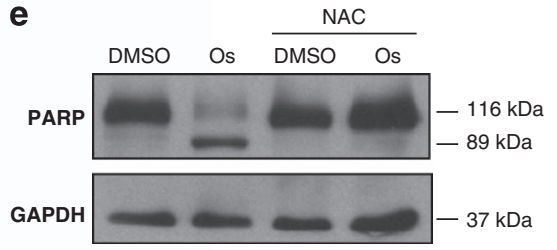

Figure 3 Os-induced cell death is ROS-dependent. (a) A total of $3 \times 10^{5} \mathrm{HCT116}$ cells were incubated with $50 \mu \mathrm{M}$ of Os for 1 or $3 \mathrm{~h}$, and intramitochondrial $\mathrm{O}_{2}^{-}$was determined using the fluorescent dye MitoSox Red and analyzed by flow cytometry. (b) Cells were preincubated with $1.5 \mathrm{mM}$ of NAC for $1.5 \mathrm{~h}$ before incubation with $50 \mu \mathrm{M}$ of Os for $4 \mathrm{~h}$, and intracellular $\mathrm{H}_{2} \mathrm{O}_{2}$ was detected by DCFH-DA loading and analyzed by flow cytometry. (c) Cells were preincubated with $1.5 \mathrm{mM}$ of NAC for $1.5 \mathrm{~h}$ before incubation with $50 \mu \mathrm{M}$ of Os for $24 \mathrm{~h}$. Cell viability was determined by crystal violet. (d) Cells were treated with NAC and Os as in (b) and 20000 cells were seeded onto 100$\mathrm{mm}$ petridishes for assessment of colony formation. (e) After preincubation with NAC and Os treatment, cell lysates were used for western blot analysis using PARP antibody. $\bullet P<0.025$ versus control

and SW620 cells (Figure 4b). Similarly, PARP cleavage was observed in RKO cells after Os treatment but not in HT29 and SW620 (Figure 4c). As both SW620 and HT29 carry a mutant form of p53, we decided to check if the death resistance could be a function of a loss of p53. Using

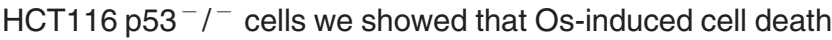
was not significantly affected by the gene knockout of p53 (Supplementary Figure S7A). Interestingly, when we assessed mitochondrial $\mathrm{O}_{2}^{-}$and $\mathrm{H}_{2} \mathrm{O}_{2}$ production in the four colon cancer cell lines, we found that after $5 \mathrm{~h}$ of Os treatment ROS production was significantly lower in HT29 and SW620 cells, compared with HCT116 and RKO cells (Figures $4 \mathrm{~d}$ and e and Supplementary Figures S6B and C). Furthermore, flow cytometry analysis in HCT116 ${\mathrm{p} 53^{-}}^{-} /^{-}$ revealed that superoxide production was not dependent on p53 status of the cell lines (Supplementary Figure S7B). These results suggest that p53 is probably not a key factor in Os-induced cell death and that ROS production has a critical role in death execution.

ROS production is upstream of caspases activation. We next wanted to determine the chronology of events triggered upon exposure of cancer cells to Os. Interestingly, neither $\mathrm{O}_{2}^{-}$nor $\mathrm{H}_{2} \mathrm{O}_{2}$ production was blocked by preincubation with the pan-caspase inhibitor z-VAD-fmk, thus indicating that ROS production was not a downstream effect of caspases activation (Figures $5 a$ and b). However, preincubation of cells with NAC inhibited Os-mediated caspase activation, thus indicating an upstream role for ROS in Os-induced death signaling (Figure $5 \mathrm{c}$ ). It should be pointed out that preincubated with NAC did not prevent Os-induced drop in mitochondrial membrane potential (Figure $5 \mathrm{~d}$ ), which suggests that Os might be targeting mitochondria in a manner independent of ROS production.

Os inhibits tumorigenesis in vivo and synergize with chemotherapy-induced apoptosis. Intrigued by the in vitro effects of Os, we next set out to investigate its ability to inhibit tumor growth in vivo. To that end, athymic, female nude mice were randomly divided into three treatment groups: control DMSO, Os $500 \mu \mathrm{g}$ (equivalent to $28 \mathrm{mg} / \mathrm{kg}$ ) and Os $1 \mathrm{mg}$ (equivalent to $56 \mathrm{mg} / \mathrm{kg}$ ), and HCT116 cells were injected subcutaneously in the flank region. The treatment regimen was delivered over the course of 3 weeks (biweekly injections) and began once the tumors had reached an average area of $50 \mathrm{~mm}^{3}$. Our results showed that animals receiving Os had significant delayed tumor growth when injected with $1 \mathrm{mg}$ of Os compared with the control group receiving DMSO (Figure 6a). The size of the tumors in Os (1 mg)-treated group was rescued to $57 \%$ of the tumor size in the control group (Figure 6b). Importantly, no overall toxicity has been shown by weight loss, as the average weight of Ostreated mice was similar to the average weight of control mice (Figure 6c). As a further evidence for the clinical relevance of these findings, we assessed the effect of 


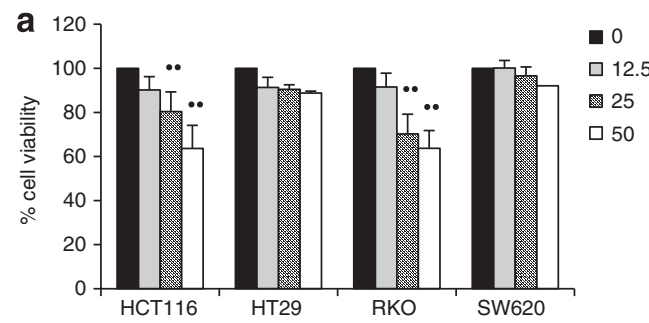

b

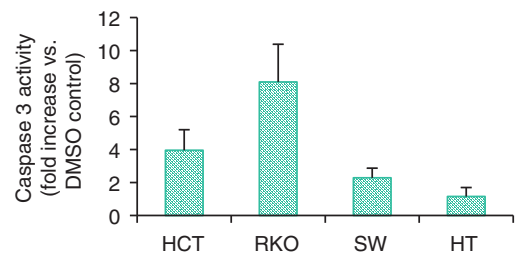

C
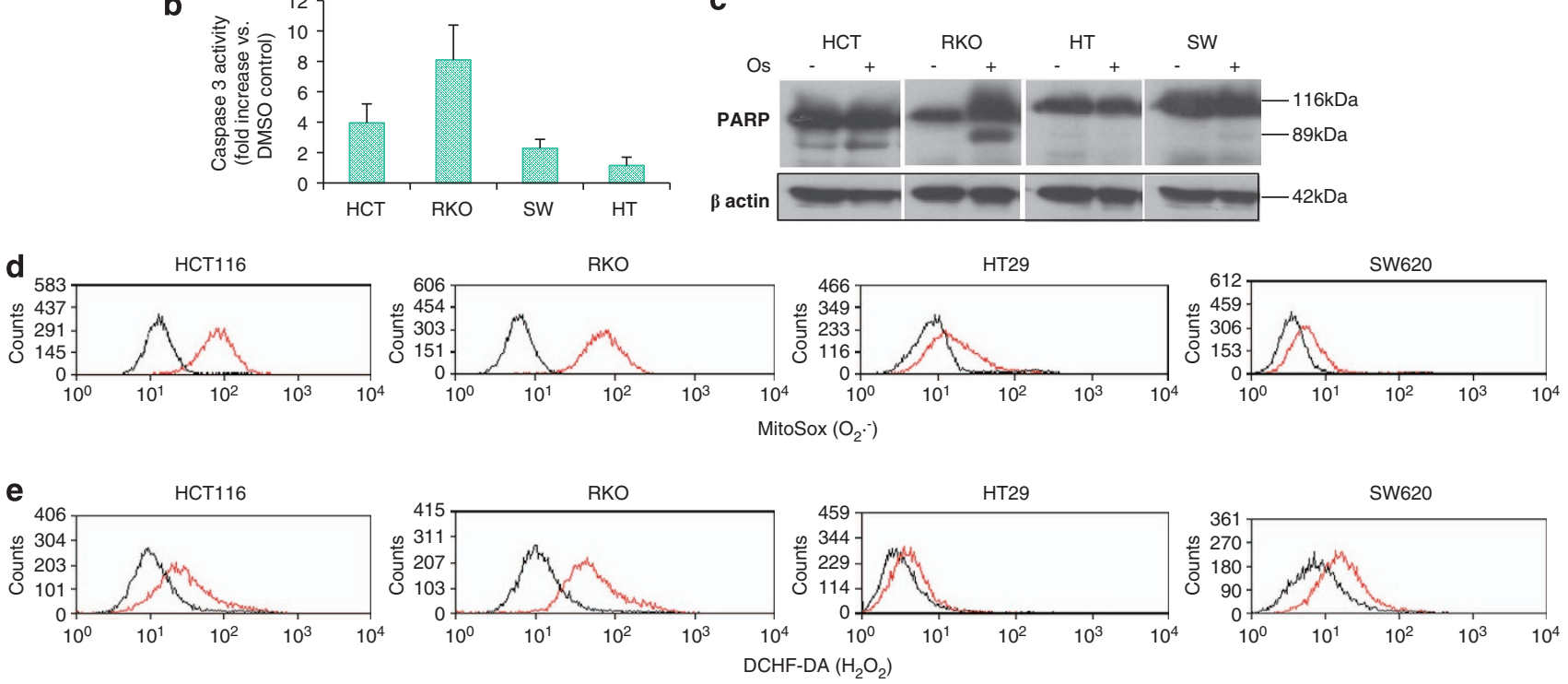

Figure 4 Colon cancer cells producing less ROS are resistant to Os-induced cell death. (a) HCT116, HT29, RKO and SW620 colon cancer cell lines were seeded in 24well plates, treated with increasing doses of Os for $24 \mathrm{~h}(12.5-50 \mu \mathrm{M})$, and cell survival was assessed by crystal violet assay. (b) Os-treated cells were harvested after $24 \mathrm{~h}$ and assayed for caspase 3 activity using fluorogenic substrates. (c) After $24 \mathrm{~h}$ of Os treatment $(50 \mu \mathrm{M})$, cells were harvested and lysates probed for processing of PARP cleavage. (d) HCT116, HT29, RKO and SW620 cells were incubated with $50 \mu \mathrm{M}$ of Os for $5 \mathrm{~h}$, intramitochondrial $\mathrm{O}_{2}^{-}$was determined using the fluorescent dye MitoSox Red (e) and intracellular $\mathrm{H}_{2} \mathrm{O}_{2}$ was detected by DCFH-DA loading (d), and cells were analyzed by flow cytometry. ${ }^{\bullet} P<0.025$ versus control

pretreatment with Os on chemotherapy-induced apoptosis. Results showed that Os treatment significantly enhanced sensitivity of HCT116 cells to cisplatin and doxorubicininduced apoptosis (Figure 6d).

Taken together, these data provide evidence that Os induces tumor cell death in colon carcinoma cells via targeting mitochondria and through ROS-dependent activation of caspases. In addition, the compound shows promise in an in vivo model of carcinogenesis and in combination therapy, which could have potential therapeutic implications.

\section{Discussion}

Os induces apoptosis by altering mitochondrial morphology and function. In this study, we report the antitumoral activity of a new organometallic drug, a novel Os-based compound, on colon cancer cell lines by a mitochondria-targeting mechanism. First, we demonstrated that Os effectively inhibited growth and long-term clonogenic survival of HCT116 and RKO colon cancer cells. Using HCT116 cells, we showed that Os-treated cells presented apoptotic features such as increase in sub-G1 population, release of cytochrome $c$, caspases 3 and 9 activation, and PARP cleavage. In addition, we observed the induction of a noncanonical autophagy prior to apoptosis induction in HCT116 cells upon Os stimulation. Indeed, Os treatment led to an increase in the conversion of LC3-I to LC3-II conversion and formation of an efficient autophagic flux in a beclin-1-independent manner. Of note, although Os-induced cell death was abrogated upon preincubation with the pan-caspase inhibitor, z-VAD-fmk, the autophagy inhibitor 3MA had no effect on the cell death process. These data are in line with previous reports demonstrating that the role of autophagy in cell death is debatable and is variable depending on the cell context. ${ }^{30,31}$

Mitochondria have a central role in intrinsic apoptotic signaling by providing amplification factors such as cytochrome $c$, Smac/Diablo and AIF, and this execution pathway is regulated by the $\mathrm{Bcl}-2$ family. ${ }^{32}$ This is generally a function of a balance between the pro-apoptotic members such as Bax and Bak, and their anti-apoptotic counterparts Bcl-2 and Bcl$\mathrm{xL}$. During apoptotic signaling Bax translocates to the mitochondria and induces MOMP, which facilitates the egress of pro-apoptotic factors for the downstream apoptosome assembly. ${ }^{33}$ On the contrary, overexpression of $\mathrm{Bcl}-2$ prevents the oligimerization of $\mathrm{Bax}$ at the mitochondria, and thereby inhibits death signaling. ${ }^{34}$ Overexpression of $\mathrm{Bcl}-2$ and/or downregulation of Bax are associated with drug 

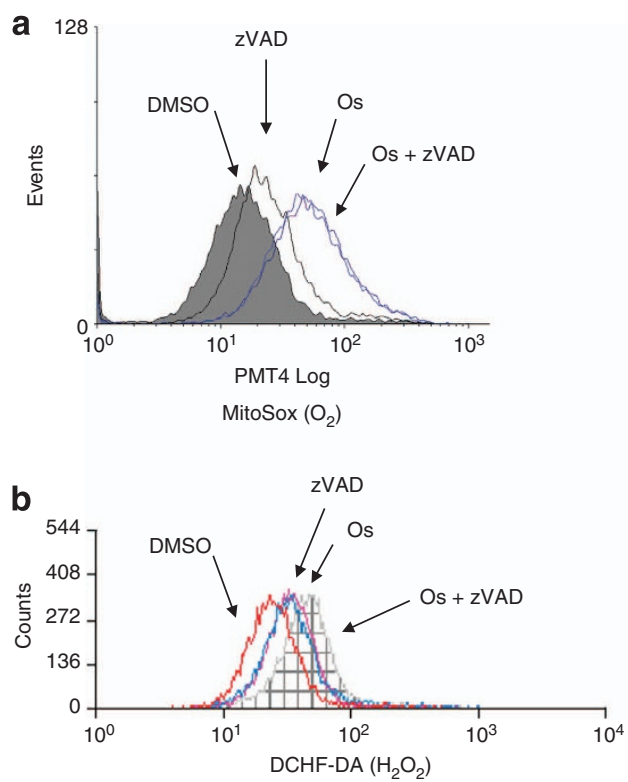
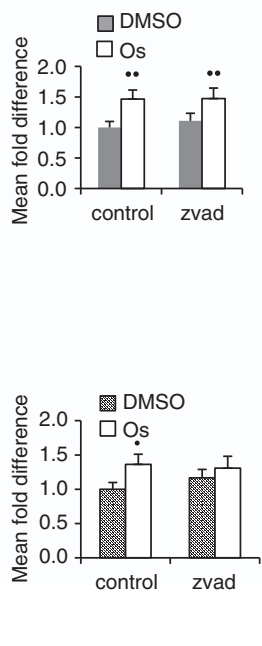

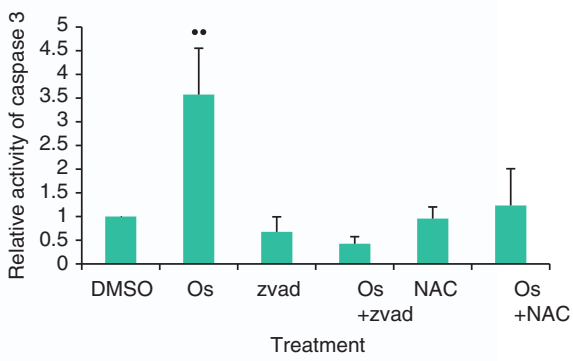

d

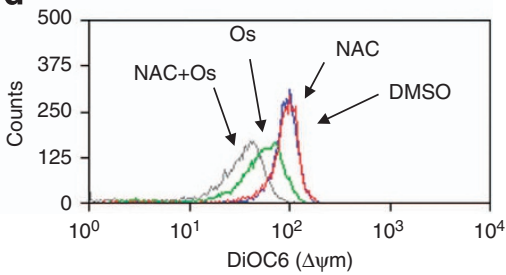

Figure 5 ROS production is upstream of caspases' activation. HCT116 cells were preincubated with ZVAD (100 $\mu \mathrm{M}$ for $1.5 \mathrm{~h})$ before exposure to Os $(50 \mu \mathrm{M})$; intramitochondrial $\mathrm{O}_{2}^{-}$was determined using the fluorescent dye MitoSox Red (a), and intracellular $\mathrm{H}_{2} \mathrm{O}_{2}$ was detected by DCFH-DA loading (b) before being analyzed by flow cytometry. Representative bar graphs were plotted with the G-mean values. (c) Cells were pretreated with $100 \mu \mathrm{M}$ of zVAD or $1.5 \mathrm{mM}$ of NAC for $1.5 \mathrm{~h}$, incubated with Os $(50 \mu \mathrm{M})$ for $24 \mathrm{~h}$ and assayed for caspase 3 activity using fluorogenic substrates. (d) HCT116 cells were preincubated with $1.5 \mathrm{mM}$ of NAC, then treated with $50 \mu \mathrm{M}$ Os for $5 \mathrm{~h}$ and loaded with the potential-sensitive probe $\mathrm{DiOC}_{6}$, and $\Delta \Psi_{\mathrm{m}}$ was analyzed by flow cytometry. ${ }^{\bullet} P<0.025$ versus control

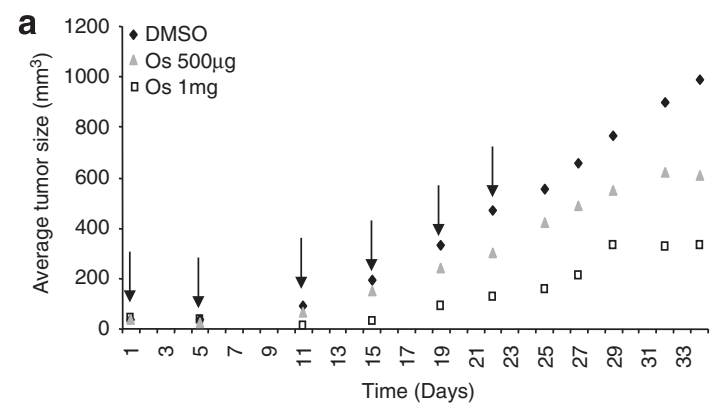

b
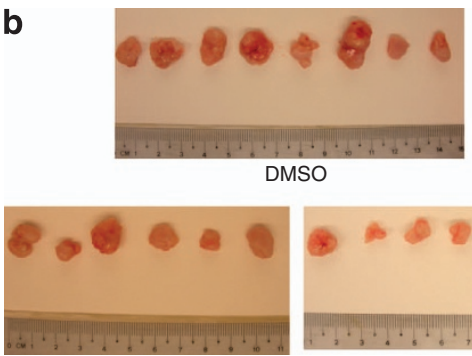

Os $500 \mu \mathrm{g}$

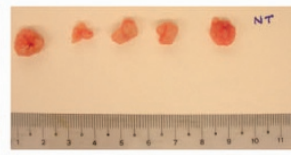

C

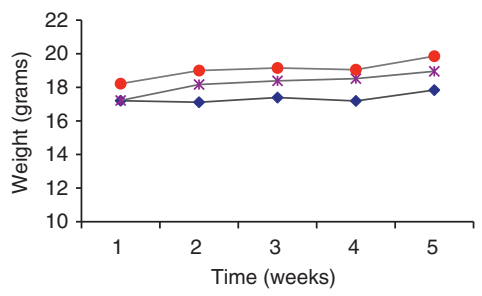

d $\quad$ drug alone

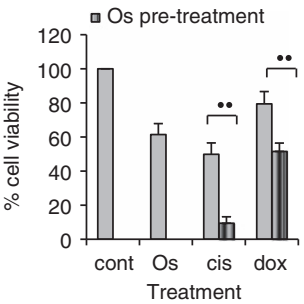

Figure 6 Os inhibits tumorigenesis in vivo and sensitizes cancer cells to cytotoxic agents. (a) Balb/c nude mice carrying HCT116 ectopic tumors (around $50 \mathrm{~mm}^{3}$ ) received intraperitoneally Os ( $500 \mu \mathrm{g}$, equivalent to $28 \mathrm{mg} / \mathrm{kg}$ ), Os (1 mg, equivalent to $56 \mathrm{mg} / \mathrm{kg}$ ) or DMSO two times a week during 3 weeks. All the mice were killed after $10 \mathrm{more}$ days of monitoring. Arrows indicate the time of injections. The results are presented as average of six animals. (b) After killing, the tumors were collected and measured. NT $=$ no tumor. (c) Mice weight was measured on a weekly basis, results represent the average weight of the six animals per group. Blue: DMSO, purple: Os $1 \mathrm{mg}$, red: Os $500 \mu \mathrm{g}$. (d) HCT116 cells $\left(1.25 \times 10^{5}\right)$ were seeded in 24-well plates and treated with either $50 \mu \mathrm{M}$ of Os alone, $50 \mu \mathrm{M}$ of cisplatin alone, $5 \mu \mathrm{M}$ of doxorubicin alone for $24 \mathrm{~h}$ or pretreated with $50 \mu \mathrm{M}$ of Os for $2 \mathrm{~h}$ and then with $50 \mu \mathrm{M}$ of cisplatin (cis), $5 \mu \mathrm{M}$ of doxorubicin (dox) for $24 \mathrm{~h}$. Cell survival was assessed by crystal violet assay. $\bullet P<0.025$ versus control

resistance in the clinical settings. ${ }^{35,36}$ Interestingly, we show here that Os triggered MOMP in a manner independent of Bax or Bcl-2, as neither the knockdown of Bax nor the overexpression of $\mathrm{Bcl}-2$ had any significant effect on its apoptotic activity. These data suggested that Os could be mediating its antitumor effect via directly targeting the mitochondria. Indeed, exposure of cells to Os resulted in altered morphology of the mitochondria, dissipation of mitochondrial membrane 
potential, decrease in mitochondrial biogenesis, as well as a significant reduction in mitochondrial ETC activity and ATP levels, and an increase in intracellular ROS production.

Critical involvement of mitochondrial ROS in Osinduced apoptosis. The cellular redox homeostasis is maintained by a tight balance between the rates of ROS production and the ability of the various antioxidant defense systems to modulate intracellular redox status. It is now well established that the cellular redox status has an important role in a variety of cellular processes such as gene expression, protein function, proliferation, cell survival and cell death. ${ }^{37,38}$ In addition to the membrane NADPH oxidase (Nox) system, mitochondria are an important source of ROS due to the high flux of electrons through the ETC that predisposes to leakage of electrons onto oxygen to generate $\mathrm{O}_{2}^{-}$. Interestingly, exposure of HCT116 cells to Os resulted in a significant increase in mitochondrial ROS production, which could be due to its ability to inhibit complex II of the ETC.

As intracellular ROS could function upstream or downstream of the mitochondria, we next set out to assess the chronology of events following Os exposure. Interestingly, ROS production was not affected by inhibiting caspase activity (z-VAD-fmk), however, preincubation of cells with the ROS scavenger NAC significantly blocked caspase 3 activation and cleavage of its substrate PARP. It should be pointed out that NAC had a minimal effect on mitochondrial morphology, thus suggesting a downstream role for ROS in Os-induced cell death. A similar role of ROS in triggering caspase cascade has been reported by earlier studies. ${ }^{39,40}$ In addition, a regulatory role for ROS in apoptosome formation and downstream caspase activation via oxidizing Apaf-1 has also been reported. ${ }^{41}$

The critical importance of mitochondrial ROS was further highlighted by our findings that the antitumor activity of Os strongly correlated with its ability to trigger mitochondrial ROS production, as HT29 and SW620 cell lines that were relatively resistant to Os (compared with HCT116 and RKO cells) showed a significantly muted response to Os in terms of ROS production. These data provide evidence that Os-induced apoptosis in human colon carcinoma cells by interfering with mitochondrial function and triggering redox-dependent activation of death execution. In this regard, triggering overproduction of ROS is one of the approaches proposed to disrupt mitochondrial functions in cancer cells. A few compounds are already under investigation, such as menadione or $\beta$-lapachone, and show promising results in preclinical studies. $^{42,43}$

Os inhibits tumor growth in a xenograft murine model. To further evaulate the clinical potential of this compound, we evaluated the ability of Os to inhibit tumor growth in a murine model of HCT116 carcinogenesis. Our results showed delayed tumor growth and a significant reduction in tumor size (43\% of vehicle-treated animals) in mice with biweekly injections of $56 \mathrm{mg} / \mathrm{kg}$ over 3 weeks. Of note, the animals did not show any signs of systemic toxicity such as weight loss or any behavioral changes throughout the duration of the study. These data corroborate the in vitro antitumor activity of this novel compound, which could have potential therapeutic implications. Although the preliminary in vivo data show promise, a thorough toxicological analysis as well as assessment of drug pharmacodynamics/pharmacokinetics need to be performed in order to gauge the true therapeutic potential of Os. Nonetheless, the in vitro antitumor activity and the preliminary in vivo data suggest that Os-based compounds might have clinical relevance. Whether the activity is comparable to the more broadly used platinumbased compounds remains to be determined.

\section{Materials and Methods}

Synthesis and analysis of Os compound. $3 \mathrm{OsO}_{4}+24 \mathrm{CO} \rightarrow$ $\left[\mathrm{Os}_{3}(\mathrm{CO})_{12}\right]+12 \mathrm{CO}_{2}$

$\mathrm{Os}_{3}(\mathrm{CO})_{12}+2\left(\mathrm{CH}_{3}\right) 3 \mathrm{NO} \rightarrow\left[\mathrm{Os}_{3}(\mathrm{CO})_{10}\left(\mathrm{NCCH}_{3}\right)_{2}\right]+2 \mathrm{~N}\left(\mathrm{CH}_{3}\right)_{3}+2 \mathrm{CO}_{2}$

Preparation of dodecacarbonyl triosmium: Osmium tetraoxide (Sigma Aldrich, St. Louis, MO, USA) and anhydrous methanol were placed in an autoclave filled with carbon monoxide. The reaction mixture was heated at $175^{\circ} \mathrm{C}$ for $10 \mathrm{~h}$. Bright-yellow crystals of dodecacarbonyl triosmium 1,2 were separated by filtration and washed with methanol. The obtained product was characterized by IR spectroscopy: IR ( $v$ $\mathrm{CO}$ ) in $\mathrm{CH}_{2} \mathrm{Cl}_{2}:$ 2068(s), 2034(s), 2013(w), 2000(w) cm-1.

Preparation of Bis(acetonitrile)decacarbonyltriosmium: A suspension of [Os3(CO)12] was prepared in acetonitrile and dichloromethane, and then heated under reflux for $1 \mathrm{~h}$. A total of $1.31 \mathrm{mmol}$ of trimethylamine oxide in acetonitrile were added. The carbonyl region of the infrared spectrum of the product obtained showed the following absorptions (cm-1, in dichloromethane) 2079(w), 2025 (s, sh), 2020(vs), $1980 \mathrm{~m}$ and 1958(w).

Preparation of $\left[\mathrm{Os}_{3}(\mathrm{CO}){ }_{10}(\mu-\mathrm{H})(\mu-\mathrm{S}) \mathrm{C}_{9} \mathrm{H}_{6} \mathrm{~N}\right]$ : $\quad\left[\mathrm{Os}_{3}(\mathrm{CO})_{10}(\mathrm{NCMe})_{2}\right]$ and 2quinolinethiol (Sigma Aldrich) were added with tetrahydrofuran. The reaction mixture was refluxed and stirred continuously under nitrogen until the starting material was completely exhausted. The residue was dissolved in dichloromethane and subjected to TLC analysis. The pure product was placed on mixture of solvents for crystal growing at $4{ }^{\circ} \mathrm{C}$. The final product, $\left[\mathrm{Os}_{3}(\mathrm{CO})_{10}(\mu-\mathrm{H})(\mu-\mathrm{S}) \mathrm{C}_{9} \mathrm{H}_{6} \mathrm{~N}\right]$, was confirmed by NMR, mass, elemental analysis IR and X-ray crystallography with $M W=1011$ (Supplementary Figure S1A).

Cell lines and reagents. Human colorectal carcinoma (HCC) cell lines $\mathrm{HCT} 116, \mathrm{HCT} 116 \mathrm{Bax}^{-} /^{-}$and HCT116 ${\mathrm{p} 53^{-}}^{-}$were generously provided by Dr. Bert Vogelstein (The Johns Hopkins University School of Medicine, Baltimore, MD, USA). HT29 and RKO colon carcinoma cell lines, as well as SW602 colorectal adenocarcinoma cell line were purchased from ATCC (Rockville, MD, USA). HCT116 and HT 29 cells were maintained in McCoy 5A (Gibco Invitrogen Corporation, Carlsbad, CA, USA), RKO cells in RPMI (Hyclone laboratories, Logan, Utah) and SW602 in DMEM (Hyclone laboratories), all medium supplemented with $1 \%$ L-glutamine, $10 \%$ fetal bovine serum and $1 \%$ S-Penicillin (Hyclone, Thermo Scientific, Waltham, MA) in a $37^{\circ} \mathrm{C}$ incubator at $5 \% \mathrm{CO}_{2}$. The pan-caspase inhibitor, z-VAD-fmk was purchased from Alexis Biochemicals (Lausen, Switzerland). The Erk inhibitor (PD98059), E64D, pepstatin-A, 3 Methyladenine, rapamycin, staurosporine, doxorubicin, cisplatin, $\mathrm{N}$-acetyl-cystein, crystal violet and MTT (3-(4,5-dimethylthiazol-2-yl)-2,5-diphenyltetrazolium bromide) were obtained from Sigma Aldrich.

Cell viability and tumor colony-formation assays. Cell viability assay was determined by crystal violet staining, as described previously. ${ }^{44,45}$ For colony-forming assays, after treatment, 10000 cells were plated in six-well plate. After 10 days, the plates were stained with crystal violet and colonies were scored manually. For siRNA experiments, cell viability was determined by the trypan blue dye exclusion test.

Propidium iodide staining for DNA fragmentation. Cells were treated with increasing doses of Os for $16 \mathrm{~h}$, fixed with $70 \% \mathrm{EtOH}$ and stained with propidium iodide staining for DNA content analysis, as described previously. ${ }^{46}$ DNA fragmentation was analyzed by flow cytometry, with the excitation and emission wavelengths at 488 and $610 \mathrm{~nm}$, respectively. At least 10000 events were analyzed by WinMDI software (designed by J. Trotter).

Assessment of caspase activity. HCT116 cells were incubated with Os $(50 \mu \mathrm{M})$ for $8,16,24$ or $48 \mathrm{~h}$. Cells were then washed with $1 \times \mathrm{PBS}$, and caspases 3,8 and 9 activities were determined by using AFC-conjugated 
substrates supplied by Bio-Rad Laboratories (Hercules, CA, USA), as previously described. ${ }^{11}$

Isolation of mitochondria. HCT116 cells treated with Os for $16 \mathrm{~h}$ were harvested and pelleted by centrifugation at $1500 \times g$ for 3 min at $4{ }^{\circ} \mathrm{C}$. Pellet was resuspended in $500 \mu \mathrm{l}$ of fractionation buffer $(250 \mathrm{mM}$ sucrose, $10 \mathrm{mM} \mathrm{KCl}$ $0.15 \mathrm{mM} \mathrm{MgCl}, 2 \mathrm{mM}$ EGTA and $1 \mathrm{mM}$ DTT) containing a cocktail of protease inhibitors, and incubated $7 \mathrm{~min}$ on ice. After incubation, cells were homogenized with a manual homogenizer for seven passages and then centrifuged at $1500 \times \mathrm{g}$ for $15 \mathrm{~min}$ at $4{ }^{\circ} \mathrm{C}$. The supernatant obtained was centrifuged at $10000 \times g$ for $15 \mathrm{~min}$ at $4^{\circ} \mathrm{C}$. The mitochondrial pellets were lysed in standard RIPA lysis buffer and the supernatants were used as the cytosolic fractions.

Western blot analysis. Os-treated cells were lysed with $100 \mu \mathrm{l}$ of RIPA buffer, equal amounts of protein were separated on SDS-PAGE (12 or $15 \%$ ) and transferred to polyvinyldene difluoride (PVDF) membranes by electroblotting. The membranes were incubated with $5 \%$ nonfat dry milk diluted in TBS-T and then incubated with primary antibodies specific for caspase 3, caspase 8, PARP, LC3, Beclin-1, Bax, CuZnSOD, CoxVa VDAC, cytochrome $c$, P-Erk, Erk (Cell Signaling Technology, Beverly, MA, USA), $\beta$ actin, p62, GAPDH, prohibitin (Santa Cruz Technology, Santa Cruz, CA, USA) or p53 (Upstate, Millipore Corporation, New York, NY, USA). The blots were then incubated with the appropriate secondary antibody conjugated to HRP. The membranes were developed using EZ-ECL (Biological Industries, Kibbutz Beit-Haemek, Israel) or Immobilon Western (Millipore, Billerica, MA, USA).

Electron microscopy. Cells were fixed overnight in $2.5 \%$ glutaraldehyde before being post-fixed in $1 \% \mathrm{OsO}_{4}$ for $1 \mathrm{~h}$. Then, cells were dehydrated in ethanol series and embedded in resin. Ultrathin sections were observed under JEOL JEM1220 transmission electron microscope (JEOL Ltd, Tokyo, Japan).

Transfection with siRNA. For knockdown of gene expression, $50 \mathrm{nM}$ of siRNA (beclin-1 siRNA, Erkl siRNA, Erkll siRNA or p53 siRNA) were transfected into cells in Optimem1 medium using the Dharmafect reagent (Dharmacon, Lafayette, CO, USA) according to the manufacturer's instructions.

Determination of intracellular ATP. HCT116 cells were treated with Os for 2, 4 or $6 \mathrm{~h}$. Subsequently, cells were resuspended in $100 \mathrm{mM}$ Tris, $4 \mathrm{mM}$ EDTA, $\mathrm{pH}$ 7.75. ATP level was determined using luciferase reagent using the ATP Bioluminescence Assay Kit CLS II (Roche, Penzberg, Germany) according to the manufacturer's instructions.

Determination of ETC complexes' activities. Quantitative analysis of human complexes I, II and IV activity was performed using the Human Complex activity Microplate Assay Kit (Mitosciences, Eugene, OR, USA). Experiments were carried out according to the protocol provided by the manufacturer.

Flow cytometric analysis of mitochondrial mass. HCT116 cells were treated for 4 or $6 \mathrm{~h}$ with $50 \mu \mathrm{M}$ of Os. Cells were then washed and incubated with NAO that binds to the inner mitochondrial membrane phospholipid cardiolipin ${ }^{47}$ for $30 \mathrm{~min}$ at $37^{\circ} \mathrm{C}$, and immediately analyzed using flow cytometry.

Determination of mitochondrial transmembrane potential $\Delta \Psi_{\mathrm{m}}$. Potential-sensitive probe $\mathrm{DiOC}_{6}$ was used to measure mitochondrial $\Delta \Psi_{\mathrm{m}}$, as described elsewhere. ${ }^{48}$ Briefly, cells were incubated with DiOC $_{6}(40 \mathrm{nM})$ for $15 \mathrm{~min}$ at $37^{\circ} \mathrm{C}$. As a positive control, cells were incubated separately with $100 \mathrm{nM}$ of cyanide m-chlorophenylhydrazone (CCCP), an uncoupling agent. Cells were analyzed in Epic Profile flow cytometer, with excitation set at $488 \mathrm{~nm}$.

Flow cytometric analysis of intracellular ROS. Intracellular concentration of $\mathrm{H}_{2} \mathrm{O}_{2}$ was determined by staining with the redox-sensitive dye 5-(and -6)chloromethyl-2-,7-dichlorofluorescin diacetate (Molecular Probes, Invitrogen Corporation). ${ }^{13}$ Briefly, after treatment, cells were washed with PBS, loaded with $5 \mu \mathrm{mol} / /$ of DCFH-DA $\left(2^{\prime}, 7^{\prime}\right.$-dichlorofluorescein diacetate) at $37^{\circ} \mathrm{C}$ for $15 \mathrm{~min}$ and analyzed by flow cytometry using an excitation wavelength of $488 \mathrm{~nm}$ (Coulter EPICS Elite ESP, Beckman Coulter, Brea, CA, USA). Detection of intramitochondrial $\mathrm{O}_{2}^{-}$was performed by loading cells with MitoSox red mitochondrial $\mathrm{O}_{2}^{-}$ indicator (Molecular Probes, Invitrogen Corporation) as described previously ${ }^{44}$ and analyzed by flow cytometry.
Assessment of in vivo antitumor activity. Female Balb/c nude mice (5 weeks old) were purchased from Animal Resources Center, Australia. Animals were handled in accordance with the guide for the care and use of laboratory animals (National Research Council 1996). Tumors were implanted in mice $\left(5 \times 10^{6} \mathrm{HCT} 116\right.$ cells), and 3 weeks later, the mice were assigned to one of the three groups; Os $(500 \mu \mathrm{g})$, Os (1 mg) or DMSO (solvent control) two times a week during 3 weeks. Mice were killed after one more week of monitoring. Their tumors were removed and frozen in liquid nitrogen.

Statistical analysis. All experiments were performed at least three times and Student's $t$-test was used for obtaining significance when comparing data sets. A $P$-value $<0.05$ was considered significant.

\section{Conflict of Interest}

The authors declare no conflict of interest.

Acknowledgements. S.P. is supported by grants from the National Medical Research Council and the Biomedical Research Council of Singapore, and the Experimental Therapeutics Program of the Cancer Science Institute, National University of Singapore, Singapore.

1. Tan ML, Ooi JP, Ismail N, Moad Al, Muhammad TS. Programmed cell death pathways and current antitumor targets. Pharm Res 2009; 26: 1547-1560.

2. Dias N, Bailly C. Drugs targeting mitochondrial functions to control tumor cell growth. Biochem Pharmacol 2005; 70: 1-12

3. Fulda S, Galluzzi L, Kroemer G. Targeting mitochondria for cancer therapy. Nat Rev Drug Discov 2010; 9: 447-464.

4. Modica-Napolitano JS, Kulawiec M, Singh KK. Mitochondria and human cancer. Curr Mol Med 2007; 7: 121-131.

5. Neuzil J, Dong LF, Rohlena J, Truksa J, Ralph SJ. Classification of mitocans, anti-cancer drugs acting on mitochondria. Mitochondrion 2012; 13: 199-208.

6. Cotter TG. Apoptosis and cancer: the genesis of a research field. Nat Rev Cancer 2009; 9 : 501-507.

7. Johnstone RW, Frew AJ, Smyth MJ. The TRAIL apoptotic pathway in cancer onset, progression and therapy. Nat Rev Cancer 2008; 8: 782-798.

8. Tait SW, Green DR. Mitochondria and cell signalling. J Cell Sci 2012; 125(Pt 4): 807-815.

9. Green DR. Apoptotic pathways: paper wraps stone blunts scissors. Cell 2000; 102: 1-4.

10. Tait SW, Green DR. Mitochondria and cell death: outer membrane permeabilization and beyond. Nat Rev Mol Cell Biol 2010; 11: 621-632.

11. Ahmad KA, Clement MV, Pervaiz S. Pro-oxidant activity of low doses of resveratrol inhibits hydrogen peroxide-induced apoptosis. Ann N Y Acad Sci 2003; 1010: 365-373.

12. Ahmad KA, Iskandar KB, Hirpara JL, Clement MV, Pervaiz S. Hydrogen peroxidemediated cytosolic acidification is a signal for mitochondrial translocation of Bax during drug-induced apoptosis of tumor cells. Cancer Res 2004; 64: 7867-7878.

13. Hirpara JL, Clement MV, Pervaiz S. Intracellular acidification triggered by mitochondrialderived hydrogen peroxide is an effector mechanism for drug-induced apoptosis in tumor cells. J Biol Chem 2001; 276: 514-521.

14. Finkel T, Holbrook NJ. Oxidants, oxidative stress and the biology of ageing. Nature 2000; 408: 239-247.

15. Wong CH, Iskandar KB, Yadav SK, Hirpara JL, Loh T, Pervaiz S. Simultaneous induction of non-canonical autophagy and apoptosis in cancer cells by ROS-dependent ERK and JNK activation. PLoS One 2010; 5: e9996.

16. Li X, Wu WK, Sun B, Cui M, Liu S, Gao J et al. Dihydroptychantol A, a macrocyclic bisbibenzyl derivative, induces autophagy and following apoptosis associated with p53 pathway in human osteosarcoma U2OS cells. Toxicol Appl Pharmacol 2011; 251: 146-154.

17. Trejo-Solis C, Jimenez-Farfan D, Rodriguez-Enriquez S, Fernandez-Valverde F, CruzSalgado A, Ruiz-Azuara $L$ et al. Copper compound induces autophagy and apoptosis of glioma cells by reactive oxygen species and JNK activation. BMC Cancer 2012; 12: 156.

18. Zhang L, Jing H, Cui L, Li H, Zhou B, Zhou G et al. 3, 4-dimethoxystilbene, a resveratrol derivative with anti-angiogenic effect, induces both macroautophagy and apoptosis in endothelial cells. J Cell Biochem 2012; 114: 697-707.

19. Kourtis N, Tavernarakis N. Autophagy and cell death in model organisms. Cell Death Differ 2009; 16: 21-30.

20. Monneret $\mathrm{C}$. Platinum anticancer drugs. From serendipity to rational design. Ann Pharm $\mathrm{Fr}$ 2011; 69: 286-295.

21. Muhlgassner G, Bartel C, Schmid WF, Jakupec MA, Arion VB, Keppler BK. Biological activity of ruthenium and osmium arene complexes with modified paullones in human cancer cells. J Inorg Biochem 2012; 116: 180-187.

22. Fu Y, Habtemariam A, Pizarro AM, van Rijt SH, Healey DJ, Cooper PA et al. Organometallic osmium arene complexes with potent cancer cell cytotoxicity. J Med Chem 2010; 53: 8192-8196. 
23. Tanida I, Minematsu-Ikeguchi N, Ueno T, Kominami E. Lysosomal turnover, but not a cellular level, of endogenous LC3 is a marker for autophagy. Autophagy 2005; 1: 84-91. 24. Green DR, Reed JC. Mitochondria and apoptosis. Science 1998; 281: 1309-1312.

25. Susin SA, Lorenzo HK, Zamzami N, Marzo I, Snow BE, Brothers GM et al. Molecular characterization of mitochondrial apoptosis-inducing factor. Nature 1999; 397: 441-446.

26. Mijaljica D, Prescott M, Devenish RJ. Mitophagy and mitoptosis in disease processes. Methods Mol Biol 2010; 648: 93-106.

27. Narendra D, Tanaka A, Suen DF, Youle RJ. Parkin is recruited selectively to impaired mitochondria and promotes their autophagy. J Cell Biol 2008; 183: 795-803.

28. Clement MV, Pervaiz S. Reactive oxygen intermediates regulate cellular response to apoptotic stimuli: an hypothesis. Free Radic Res 1999; 30: 247-252.

29. Le Bras M, Clement MV, Pervaiz S, Brenner C. Reactive oxygen species and the mitochondrial signaling pathway of cell death. Histol Histopathol 2005; 20: 205-219.

30. Debnath J, Baehrecke EH, Kroemer G. Does autophagy contribute to cell death? Autophagy 2005; 1: 66-74.

31. Maiuri MC, Zalckvar E, Kimchi A, Kroemer G. Self-eating and self-killing: crosstalk between autophagy and apoptosis. Nat Rev Mol Cell Biol 2007; 8: 741-752.

32. Kluck RM, Bossy-Wetzel E, Green DR, Newmeyer DD. The release of cytochrome c from mitochondria: a primary site for Bcl-2 regulation of apoptosis. Science 1997; 275: 1132-1136.

33. Ghibelli L, Diederich M. Multistep and multitask Bax activation. Mitochondrion 2010; 10: 604-613.

34. Estaquier J, Vallette F, Vayssiere JL, Mignotte B. The mitochondrial pathways of apoptosis. Adv Exp Med Biol 2012; 942: 157-183.

35. Kassim SK, Ali HS, Sallam MM, Fayed ST, Seada LS, abd-Elkawy E et al. Increased bcl-2 expression is associated with primary resistance to chemotherapy in human epithelial ovarian cancer. Clin Biochem 1999; 32: 333-338.

36. Wong FY, Liem N, Xie C, Yan FL, Wong WC, Wang L et al. Combination therapy with gossypol reveals synergism against gemcitabine resistance in cancer cells with high $\mathrm{BCL}-2$ expression. PLoS One 2012; 7: e50786.

37. Burhans WC, Heintz NH. The cell cycle is a redox cycle: linking phase-specific targets to cell fate. Free Radic Biol Med 2009; 47: 1282-1293.

38. Halliwell B, Gutteridge JM, Cross CE. Free radicals, antioxidants, and human disease: where are we now? J Lab Clin Med 1992; 119: 598-620.

39. Higuchi M, Honda T, Proske RJ, Yeh ET. Regulation of reactive oxygen species-induced apoptosis and necrosis by caspase 3-like proteases. Oncogene 1998; 17: 2753-2760.
40. Zuo Y, Xiang B, Yang J, Sun X, Wang Y, Cang H et al. Oxidative modification of caspase-9 facilitates its activation via disulfide-mediated interaction with Apaf-1. Cell Res 2009; 19: 449-457.

41. Sato T, Machida T, Takahashi S, lyama S, Sato Y, Kuribayashi K et al. Fas-mediated apoptosome formation is dependent on reactive oxygen species derived from mitochondrial permeability transition in Jurkat cells. J Immunol 2004; 173: 285-296.

42. Bey EA, Bentle MS, Reinicke KE, Dong Y, Yang CR, Girard L et al. An NQO1- and PARP1 -mediated cell death pathway induced in non-small-cell lung cancer cells by betalapachone. Proc Natl Acad Sci USA 2007; 104: 11832-11837.

43. Lim D, Morgan Jr RJ, Akman S, Margolin K, Carr BI, Leong L et al. Phase I trial of menadiol diphosphate (vitamin K3) in advanced malignancy. Invest New Drugs 2005; 23: 235-239.

44. Low IC, Chen ZX, Pervaiz S. Bcl-2 modulates resveratrol-induced ROS production by regulating mitochondrial respiration in tumor cells. Antioxid Redox Signal 2010; 13: 807-819.

45. Shenoy K, Wu Y, Pervaiz S. LY303511 enhances TRAIL sensitivity of SHEP-1 neuroblastoma cells via hydrogen peroxide-mediated mitogen-activated protein kinase activation and up-regulation of death receptors. Cancer Res 2009; 69: 1941-1950.

46. Clement MV, Ponton A, Pervaiz S. Apoptosis induced by hydrogen peroxide is mediated by decreased superoxide anion concentration and reduction of intracellular milieu. FEBS Lett 1998; 440: 13-18

47. Tang $Y$, Chen $Y$, Jiang $H$, Nie D. Short-chain fatty acids induced autophagy serves as an adaptive strategy for retarding mitochondria-mediated apoptotic cell death. Cell Death Differ 2011; 18: 602-618.

48. Clement MV, Hirpara JL, Pervaiz S. Decrease in intracellular superoxide sensitizes Bcl-2overexpressing tumor cells to receptor and drug-induced apoptosis independent of the mitochondria. Cell Death Differ 2003; 10: 1273-1285.

(c) (i) $\odot$ Cell Death and Disease is an open-access journal published by Nature Publishing Group. This work is licensed under a Creative Commons Attribution-NonCommercialNoDerivs 3.0 Unported License. To view a copy of this license, visit http://creativecommons.org/licenses/by-nc-nd/3.0/

Supplementary Information accompanies this paper on Cell Death and Disease website (http://www.nature.com/cddis) 